\title{
Comparison of the Effect of Oral Diphenhydramine and Midazolam on Sedation of Children
}

\author{
Neda Naeimi Bafghi, Naeimeh Naeimi Bafghi*, Shirin Salajegheh
}

Clinical Research Center, Shahid Bahonar Hospital, Kerman University of Medical Sciences, Kerman, Iran.

*Correspondence to: Naeimeh Naeimi Bafghi (E-mail: NaeimehNaeimiBafghi@yahoo.com)

(Submitted: 14 April 2021 - Revised version received: 02 May 2021 - Accepted: 29 May 2021 - Published online: 26 August 2021)

\begin{abstract}
Objective The aim of this study was to compare the effect of diphenhydramine and midazolam on sedation of children.

Methods This clinical trial was performed on children aged 1 to 7 years who referred to the emergency department for diagnostic radiology. Patients were randomly divided into two groups of midazolam and diphenhydramine. Then, 30 minutes before the start of the procedure, $0.5 \mathrm{mg} / \mathrm{kg}$ was given to the midazolam group and $0.5 \mathrm{cc} / \mathrm{kg}$ to the diphenhydramine group. If sedation occurred, the child was separated from the parents and transferred to a diagnostic procedure. After performing the intended diagnostic procedure, the information sheet was completed and the patient's vital signs were checked again. The data were then analyzed by SPSS version 19 software.

Results A total of 74 patients were included in the study. There was no significant difference between the two groups in terms of age and gender $(P=0.89 ; P=0.32$ ). The mean sedation in the midazolam and diphenhydramine groups was 1.02 and 1.59 years, respectively. A significant difference was found between the two groups in terms of sedation $(P=0.04)$, where a greater effect of diphenhydramine on sedation was observed.

Conclusion The findings showed that the use of diphenhydramine resulted in effective sedation for children. Due to the fact that the main problem with midazolam is its bitter taste, which makes children reluctant to eat it, the use of diphenhydramine can be recommended.

Keywords Diphenhydramine, midazolam, sedation
\end{abstract}

\section{Introduction}

Fear and anxiety before a diagnostic-therapeutic process or before anesthesia and surgery can be a more traumatic experience than the process itself for many patients, especially children. ${ }^{1,2}$

According to studies, nothing could even replace parental support and caress in many cases, but this support is not effective in relieving preoperative fear and excitement, thus prodrug has a significant clinical effect in reducing the child's harm from anesthesia and surgery. ${ }^{3,4}$ Studies show that parental anxiety make it more difficult to separate children. Some studies have also shown that prescribing sedatives before surgery prevent adverse postoperative reactions such as nocturnal enuresis and anorexia. Numerous studies have shown that almost all sedatives are effective as prodrugs, ${ }^{6}$ However, the selection of an appropriate prodrug should be done by considering the desired amount of sedation on the one hand and the effectiveness, side effects and indications of each drug on the other hand.

The need for prodrug varies depending on the patient's condition, underlying disease, type and duration of surgery, method of induction, and mental structure of the child and his family. Children under 8 months of age rarely need prodrugs, but after this age the child's normal development causes fear of people and unfamiliar environment. ${ }^{3-5}$ Preoperative drug administration is widely used for sedation and anti-anxiety effects in pediatric anesthesia. ${ }^{7}$ A good sedative is easy to use, rapid onset of action, short duration of action and lack of side effects. ${ }^{8}$ The prodrug used is enough to have only a sedative effect. On the other hand, prodrugs can be given by different routes oral, nasal, intravenous, or intramuscular, and rectal routs. Certainly, oral and nasal routs are more acceptable and simpler in children and are not accompanied by pain and anxiety.

The most commonly used drugs in children are diphenhydramine, dextromethorphan and midazolam. In the United States, midazolam has been produced as Versed Syrup in recent years, but this product is only available in the United States. ${ }^{9}$ Midazolam is a good sedative that can be prescribed in several routs (oral, injectable, nasal and rectal). ${ }^{10}$ The onset of action is within 10 to 20 minutes and the duration of action is 30 minutes. Midazolam at doses less than $0.5 \mathrm{mg} / \mathrm{kg}$ do not result in a change in hemodynamics ${ }^{7}$ and provides excellent sedation in 60 to $80 \%$ of patients. ${ }^{1}$

In the oral rout, the time to reach the peak effect is one hour and the reversal of the action of drug is long (up to about 4 hours), while the depth of sedation also varies. For this reason, the intranasal rout, which reaches the peak effect of the drug within ten minutes and the reversal time of the effect is not more than one hour, seems more acceptable. ${ }^{12}$

Diphenhydramine has also been used as an inverse agonist of the histamine $\mathrm{H} 1$ receptor in a variety of allergic and psychiatric diseases. It is also used as an adjunct to insomnia or sleep disorders. The use of diphenhydramine in anesthesia is limited. ${ }^{13}$ Therefore, the aim of this study was to evaluate the effect of oral diphenhydramine and midazolam on sedation of children referred to the hospital for imaging.

\section{Materials and Methods}

This clinical trial was performed on children aged 1 to 7 years who referred to the emergency department of Bahonar Hospital in Kerman, Iran, for diagnostic radiology procedures 
from May to August 2019. Patients who met the inclusion and exclusion criteria were included in the study.

Inclusion criteria: age of children between 1 to 7 years and level of consciousness in the mild-moderate range. Exclusion criteria include: parental dissatisfaction, GCS $\leq 8$ and unstable hemodynamics (lack of hemodynamics).

\section{Sample Size}

Taking into account the first and second type errors, the sample size of 74 people was calculated using the formula of comparing the two means.

$$
n_{1}=n_{2}=\frac{\left(S_{1}^{2}+S_{2}^{2}\right)\left(Z_{1-\frac{\alpha}{2}}+Z_{1-\beta}\right)^{2}}{\left(\bar{X}_{1}-\bar{X}_{2}\right)^{2}}
$$

\section{Procedure}

After obtaining informed consent, patients were randomly divided into two groups of midazolam and diphenhydramine. Then, 30 minutes before the start of the procedure, the drug was administered to the midazolam group at $0.5 \mathrm{mg} / \mathrm{kg}$ and to the diphenhydramine group at $0.5 \mathrm{cc} / \mathrm{kg}$. If sedation occurred, the child was separated from the parents and transferred to a diagnostic procedure (CT, radiology, and ultrasound). After performing the diagnostic procedure, the information sheet was completed and the patient's vital signs were checked again and children were then returned to the parents if the child was found to be healthy.

In fact, the child was resuscitated if there were unstable vital signs. The child was returned to the parents if he or she had stable vital signs.

The measurement of effectiveness criteria was as follows: The patient is sedated to the extent of Mild (Minimal)Moderate. This means that patients have a purposeful response to verbal or physical stimulation after receiving the substance by the above methods, and the airway, respiration, blood pressure and pulse are not disturbed. Reaching or not reaching this amount in each of the studied methods was considered as outcome. Sedation rates were assessed based on the the University of Michigan Sedation Scale (UMSS) with patient questioning and examination. UMSS is capable of evaluating the level of changes on a five-point scale as follow:

1. sleepy / responds UMSS: O: a wake aurt

2. somnolent / arouses to light stimuli

3. deep sleep / arouses to deporplynsical stimuli

4. unarousable to stimuli

\section{Data Analysis}

Independent $t$-test was used to compare the parameters in two independent groups. Chi-square test was used to examine qualitative variables. Data analysis was performed using SPSS software version 19 using statistical methods including descriptive and inferential statistics. A $P$ value of 0.05 was considered to be statistically significant.

\section{Ethical Considerations}

A written letter of introduction to research centers was received from university officials. The purpose of the study was described for all research units and finally written consent was obtained. All patients' information was kept confidential.
The Declaration of Helsinki was considered in the current study. The statements of the research ethics committees of the University of Medical Sciences were taken into account. The study was carried out after approval by the research council of the medical school and receiving the code (IR.KMU. REC.1397.520) of ethics letter of introduction.

\section{Results}

A total of 74 patients were included in the study. The study population consisted of 39 female patients and 35 male patients who were divided into two groups of 37 patients. There was no significant difference between the two groups in terms of gender $(P=0.32)$ (Table 1$)$.

The mean age in the diphenhydramine group was 2.89 years and the mean age was 2.83 years in the midazolam group (Table 2). No significant difference was found between the two groups in terms of age $(P=0.89)$, indicating that the groups were the same at the beginning of the study and elimination of age effects.

The mean sedation is given in Table 3, which was determined to be 1.02 and 1.59 years in the midazolam and diphenhydramine groups, respectively. There was a statistically significant difference between the two groups $(P=0.04)$ so that a higher mean in the group of diphenhydramine indicates a greater effect of diphenhydramine in sedation.

\section{Discussion}

Prodrug administration in children using a safe method and appropriate dose is very important to achieve adequate sedation. ${ }^{14}$ The aim of this study was to compare the effect of diphenhydramine and oral midazolam on sedation of children. In this study, 74 children aged 1 to 7 years were examined. Then, the findings of this study were analyzed and the final conclusion was presented.

\begin{tabular}{lcccc}
\multicolumn{4}{l}{ Table 1. Frequency distribution of subjects by gender } \\
\hline Variable & Female & Male & Total & P-value \\
\hline Diphenhydramine & $21(56.8 \%)$ & $16(43.2 \%)$ & 37 & 0.32 \\
Midazolam & $18(48.6 \%)$ & $19(51.4 \%)$ & 37 & \\
Total & 39 & 35 & 74 & \\
\hline
\end{tabular}

Table 2. Mean and standard deviation of age in the two groups

\begin{tabular}{lcccc}
\hline Variable & Mean & SD & $\boldsymbol{t}$ & $\boldsymbol{P}$-value \\
\hline Diphenhydramine & 2.89 & 1.86 & 0.13 & 0.89 \\
Midazolam & 2.83 & 1.7 & & \\
\hline
\end{tabular}

Table 3. Mean and standard deviation of sedation after intervention

\begin{tabular}{lcccc}
\hline Variable & Mean & SD & $\boldsymbol{t}$ & $\boldsymbol{P}$-value \\
\hline Diphenhydramine & 1.59 & 1.11 & 2.07 & 0.04 \\
Midazolam & 1.02 & 1.23 & & \\
\hline
\end{tabular}


In the present study, there was no significant difference between the two groups in terms of gender and age. As a result, the confounding effect of these variables has been controlled. The most important finding of this study was the significant difference between the mean sedation in the midazolam and diphenhydramine groups, where a higher mean sedation was observed in the diphenhydramine group. Cengiz et al. compared the safety and efficacy of midazolam-diphenhydramine combination and midazolam alone in pediatric sedation for magnetic resonance imaging. The results showed that the combination of oral diphenhydramine with oral midazolam is safe and effective in performing MRI in children and its sedative failure is less during MRI. ${ }^{15}$ Findings of Golzari et al.'s study also showed that the combination of diphenhydramine and midazolam has a higher sedative effect and less side effects compared to diphenhydramine alone. ${ }^{16}$ In Heydarian's research, the findings showed that the combination of oral diphenhydramine and oral midazolam leads to safe and effective sedation of children during CT scan. This combination can be more beneficial than midazolam alone. ${ }^{17}$ The results of a study by Taghipor et al., which examined the sedative effect of three oral prodrugs (midazolam, dextromethorphan and diphenhydramine) in children, indicated that the rate of sedation before any intervention in the dextromethorphan group was significantly better.

At the time of separation of children from parents, the three groups did not differ significantly in the intensity of sedation. However, the intensity of sedation in patients receiving dextromethorphan was significantly better than that of oral diphenhydramine and midazolam during induction of anesthesia and in the recovery.

In some other studies, the effect of midazolam alone in pediatric sedation has been investigated. In 2008, Lane et al. examined the use of intranasal midazolam for minor procedures in children, stating that intranasal midazolam is very suitable for providing anxiolysis reducing anxiety to children undergoing minor procedures in the pediatric department. ${ }^{19}$
In 2015, Plum et al. examined the effect of intranasal midazolam in reducing anxiety in children with nasal fractures. The results showed that midazolam was quite effective in providing effective anxiolysis and had no adverse outcomes. ${ }^{20}$

In a 2015 study, Musani et al. Concluded that intravenous midazolam had a quick onset of action and a quick recovery from sedation. Also, its proper effect required a lower dose through the intranasal route. Finally, they concluded that intranasal midazolam is a suitable alternative to oral midazolam for a pediatric dental condition. ${ }^{2}$

Many diagnostic and therapeutic measures in children require the full cooperation of the child. These diagnostic procedures include radiology, endoscopy, colonoscopy, aspiration, bone marrow biopsy, liver and kidney biopsy, bronchoscopy, and cerebrospinal fluid biopsy. The use of sedatives for diagnostic, biopsy, or minor surgery in children is a high priority. Issues such as unfamiliarity with the ward, not realizing the importance of the issue, separation from parents will lead to poor cooperation of children. Therefore, it should be tried that in the process of prescribing sedatives, drugs with ease of use, rapid onset of action, minimal side effects and more short recovery should be prescribed. Non-injectable prescriptions are highly acceptable, although they have a longer onset of action than the injectable form and have a higher initial liver removal; also, it is possible that interpersonal differences in effect rate and rate of absorption may impair the process. Sedation measures increase the quality of diagnosis and treatment and can reduce the psychological effects of the child.

\section{Conclusion}

The results of this study showed that the use of diphenhydramine is suitable for sedation and reducing anxiety in children. Due to the fact that the main problem with midazolam is its bitter taste, which makes children reluctant to eat it, the use of diphenhydramine can be recommended.

\section{References}

1. Song JH. Procedural sedation and analgesia in children. J Korean Med Assoc 2013;56(4):271-8

2. Eskandarian T, Maghsoudi S, Eftekharian H. Clinical evaluation of the effects of two types of oral combination of midazolam in sedating pediatric dental patients. J Dent Shiraz Univ Med Sci. 2010;11(1).

3. Soleimanpour H, Gholipouri C, Salarilak S, Raoufi P, Vahidi RG, Rouhi AJ, et al. Emergency department patient satisfaction survey in Imam Reza Hospital, Tabriz, Iran. Int J Emerg Med. 2011;4:2

4. Barkan S, Breitbart R, Brenner-Zada G, et al. A double-blind, randomised, placebo-controlled trial of oral midazolam plus oral ketamine for sedation of children during laceration repair. Emerg Med J. 2013;31(8):649-53.

5. Hosseini M, Karami Z, Janzadenh A, et al. The Effect of Intratheca Administration of Muscimol on Modulation of Neuropathic Pain Symptoms Resulting from Spinal Cord Injury; an Experimental Study. Emergency. 2014; 2(4):151-7

6. Alimohammadi H, Shojaee M, Samiei M, Abyari S, Vafaee A, Mirkheshti A. Nerve stimulator guided axillary block in painless reduction of distal radius fractures; a randomized clinical trial. Emergency. 2013;1(1):11-4.

7. Azizkhani R, Esmailian M, Golshani K. Rectal Thiopental versus Intramuscular Ketamine in Pediatric Procedural Sedation and Analgesia; a Randomized Clinical Trial. Emergency. 2014:3(1):22-6.

8. Khajavi M, Emami A, Etezadi F, Safari S, Sharifi A, Moharari RS. Conscious sedation and analgesia in colonoscopy: Ketamine/propofol combination has superior patient satisfaction versus fentanyl/propofol. Anesthesiol Pain Med. 2013;3(1):208-12.

9. Alimohammadi H, Azizi M-R, Safari S, Amini A, Kariman H, Hatamabadi HR Axillary Nerve Block in Comparison with Intravenous Midazolam/Fentanyl

for Painless Reduction of Upper Extremity Fractures. Acta Med Iranica. 2014: 52(2):122-4.

10. Krauss BS, Krauss BA, Green SM. Procedural sedation and analgesia in children. N Engl J Med. 2014;370(15):e23

11. Moreira TA, Costa PS, Costa LR, et al. Combined oral midazolam-ketamine better than midazolam alone for sedation of young children: a randomized controlled trial. Int J Paediatr Dent. 2013;23(3):207-15.

12. Maurizi P, Russo I, Rizzo D, et al. Safe lumbar puncture under analgosedation in children with acute lymphoblastic leukemia. Int J Clin Oncol. 2014;19(1):173-7.

13. Green SM. What is the role of diphenhydramine in local anesthesia? AcadEmerg Med. 1996:3(3):198-200

14. Alimohammadi H, Baratloo A, Abdalvand A, Rouhipour A, Safari S. Effects of Pain Relief on Arterial Blood O2 Saturation. Trauma mon. 2014;19(1):e14034.

15. Cengiz M, Baysal Z, Ganidagli S. Oral sedation with midazolam and iphenhydramine compared with midazolam alone in children undergoing magnetic resonance imaging. Pediateric Anesthesia 2006;16(6): 621-626.

16. Golzari S, Shahsavari Nia K, Sabahi M, Soleimanpour H, Mahmoodpoor A, Safari S, et al. Oral Diphenhydramine-Midazolam Versus Oral Diphenhydramine for Pediatric Sedation in the Emergency Department. J ComprPed 2014 February;5(1):e17946.

17. Heydarian N. Comparison of the simultaneous use of midazolam and oral diphenhydramine with oral midazolam in pediatric patients 1 to 7 years old at the time of CT scan referring to the emergency department of Besat Hospital. Army University of Medical Sciences, Thesis. 2014 
18. Taghipor Anvari Z, Sheibani S, Imani F, Sheibani S. Comparison of three Oral Premedication Dextrometorphan, Diphenhydramine and Midazolam in Pediatric Eye Exam under Anesthesia. JAP. 2011;2(3):9-17.

19. Lane RD, Schunk JE. Atomized intranasal midazolam use for minor procedures in the pediatric emergency department. Pediatr Emerg Care. 2008 May; 24(5):300-3.
20. Plum AW, Harris TM. Intranasal midazolam for anxiolysis in closed reduction of nasal fractures in children. Int J Pediatr Otorhinolaryngol. 2015 Jul; 79(7):1121-3

21. Musani IE, Chandan NV. A comparison of the sedative effect of oral versus nasal midazolam combined with nitrous oxide in uncooperative children. Eur Arch Paediatr Dent. 2015 May 5.

This work is licensed under a Creative Commons Attribution-NonCommercial 3.0 Unported License which allows users to read, copy, distribute and make derivative works for non-commercial purposes from the material, as long as the author of the original work is cited properly. 